\title{
BMJ Open Prevalence of stroke and associated risk factors: a population based cross sectional study from northeast China
}

\author{
Fu-Liang Zhang, ${ }^{1}$ Zhen-Ni Guo, ${ }^{2}$ Yan-Hua Wu, ${ }^{3}$ Hao-Yuan Liu, ${ }^{1}$ Yun Luo, ${ }^{1}$ \\ Ming-Shuo Sun, ${ }^{1}$ Ying-Qi Xing, ${ }^{2}$ Yi Yang ${ }^{1}$
}

To cite: Zhang F-L, Guo Z-N Wu Y-H, et al. Prevalence of stroke and associated risk factors: a population based cross sectional study from northeast China. BMJ Open 2017;7:e015758. doi:10.1136/ bmjopen-2016-015758

- Prepublication history for this paper is available online. To view these files please visit the journal online (http://dx.doi org/10.1136/bmjopen-2016015758).

Received 28 December 2016 Revised 28 May 2017 Accepted 25 July 2017

\section{(a) CrossMark}

${ }^{1}$ Department of Neurology, Stroke Centre, The First Hospital of Jilin University, Chang Chun, China

${ }^{2}$ Department of Neurology, Neuroscience Centre, The First Hospital of Jilin University, Chang Chun, China

${ }^{3}$ Division of Clinical Research, The First Hospital of Jilin University, Changchun, China

Correspondence to

Professor Yi Yang;

doctoryangyi@163.com

\section{ABSTRACT}

Objectives Epidemiological studies aimed at stroke and its risk factors can help identify persons at higher risk and therefore promote stroke prevention strategies. We aimed to explore the current prevalence of stroke and its associated risk factors in northeast China.

Design Population based cross sectional study.

Setting Data were collected using a structured precoded questionnaire designed by the Stroke Screening and Prevention Programme of the National Health and Family Planning Commission of China, between January and March 2016.

Participants 4100 permanent residents, aged 40 years or older, who had lived in Dehui City of Jilin Province for more than 6 months volunteered to participate in the survey, with a response rate of $92.2 \%$. For the purpose of the present analysis, 48 subjects were excluded due to missing values, giving a total of 4052 people included in this analysis.

Main outcome measure The questionnaire included demographic characteristics, stroke related behavioural factors, personal and family medical history of stroke, physical examination and laboratory testing.

Results The overall prevalence of stroke in Jilin Province was $7.2 \%$ (95\% Cl 6.3\% to 8.2\%). Of all stroke cases, $91.7 \%$ (95\% Cl $87.4 \%$ to $94.6 \%)$ were ischaemic stroke and $8.3 \%(95 \% \mathrm{Cl} 5.4 \%$ to $12.6 \%)$ were haemorrhagic stroke. The prevalence rates of dyslipidaemia, smoking and hypertension were ranked as the top three cerebrovascular risk factors and were $62.1 \%, 61.8 \%$ and $57.3 \%$, respectively. We found that hypertension, dyslipidaemia and lack of exercise were associated with ischaemic stroke. However, only hypertension $(0 \mathrm{R}=4.064$, $95 \% \mathrm{Cl} 1.358$ to 12.160 ) was significantly associated with haemorrhagic stroke.

Conclusions The prevalence of stroke, especially ischaemic stroke, and associated cerebrovascular risk factors among adults aged 40 years or older in northeast China were high. A higher regional prevalence of hypertension, dyslipidaemia and lack of exercise may be responsible.

\section{INTRODUCTION}

The latest data from the Global Burden of Diseases Study 2013 (GBD 2013) ranked cerebrovascular disease as the second largest contributor to death and disability adjusted

\section{Strengths and limitations of this study}

- This study was a population based cross sectional study, representing a large sample from northeast China ( $\mathrm{n}=4052)$.

- A multistage stratified random cluster method was used in the sampling process and complex weighted computation was used in the data analysis to make the result more reliable.

- There was an excellent response rate and a low percentage of missing data in general.

- Limitations of the study were the properties of the cross sectional study and recall bias of the selfreported questionnaire.

- Another limitation was that the respondents' atrial fibrillation status was based on self-report and ordinary ECG, which may be underestimated for paroxysmal atrial fibrillation.

life years worldwide after ischaemic heart disease. ${ }^{12}$ In the past several decades in developed countries, a greater reduction in the age standardised stroke incidence has taken place because of good health services and effective strategies for cerebrovascular risk factor prevention. However, the converse has been shown for developing countries. ${ }^{3}$

Despite the advent of reperfusion therapies, such as intravenous tissue type plasminogen activator and endovascular therapy, for selected patients with acute ischaemic stroke, ${ }^{4}$ there is still a proportion of patients with residual disability or cognitive deficits. Therefore, effective prevention, especially primary prevention, remains the best strategy for reducing the burden of stroke. ${ }^{56}$

Within the global stroke belt, China's stroke burden mirrors the situation seen in many developing countries. ${ }^{7}$ According to a report from the WHO, the incidence of stroke in China is still increasing at an annual rate of $8.7 \%$, and has now become the leading cause of death in China. ${ }^{8}$ In Jilin, a province of intermediate economic development located in the central part of northeast China, and 
within the China stroke belt, the stroke incidence was nearly twice that of other regions. ${ }^{10}$ However, the most widely cited data on stroke gathered by epidemiological investigation in Jilin Province were collected in the 1980s and 1990s. ${ }^{11-13}$ The Chinese lifestyle has changed greatly, and the ageing population has increased during the past few decades, which has led to changes in the prevalence of stroke and its associated risk factors. This study aims to estimate the prevalence of stroke and the pattern of its related risk factors in northeast China.

\section{METHODS}

\section{Study design and participants}

This population based cross sectional study was part of the Stroke Screening and Prevention Programme of the National Health and Family Planning Commission of China, which was one of the National Key Technology Research and Development Programmes in China (grant No. 2011BAI08B01) and supervised by the Chinese National Centre for Stroke Care Quality Control and Management. ${ }^{14} 15$ The main aims of the survey were to: (1) obtain updated and reliable data for the incidence, prevalence and mortality of stroke in different regions of China; and (2) access relevant data on stroke, including risk factors, treatment and secondary prevention. Stroke is usually thought of as a disease of the elderly, and the cumulative effects of ageing on the cardiovascular system and the progressive nature of stroke risk factors over a prolonged period substantially increase the risks of both ischaemic stroke and intracerebral haemorrhage. Because the stroke incidence and prevalence rates showed a steep increase after the age of 39 years,${ }^{16}$ the Stroke Screening and Prevention Project Committee, National Health and Family Planning Commission of China, targeted residents aged 40 years or older as the screening population. ${ }^{15}$

From January to March 2016, we conducted a cross sectional study in Dehui City, which is located in the north central part of Jilin Province. The rationale for this period of time was that our team spent 3 months performing this cross sectional survey. In contrast, the stroke prevalence rate in our survey referred to the lifetime prevalence by the end of 2015, and new onset stroke cases during this 3 month survey were not counted to avoid the influence of seasonal factors on the stroke prevalence. Finally, the reason for choosing January to March was to increase the response rate and reduce sample selection bias, making the study sample representative of adults aged 40 years or older as many migrant workers living in this area would return home for Spring Festival. Multistage stratified random cluster sampling was used to select permanent residents aged 40 years or older who had lived in this area for more than 6 months. In the first stage, 30 villages and 10 towns were selected from 308 villages (rural) and 14 towns (urban), respectively. In the second stage, cluster sampling was used in the selected areas. Respondents were interviewed in person using a structured precoded questionnaire designed by the Stroke Screening and Prevention Programme of the National Health and Family Planning Commission of China. The investigators had at least 5 years of education in medicine. They had been uniformly trained and passed the examination at the end of training.

\section{Sampling size}

According to the requirements of the Stroke Screening and Prevention Project Committee of the National Health and Family Planning Commission of China, screening should cover at least $1 \%$ of the local residents aged 40 years or older. There were 335490 residents aged 40 years or older in Dehui City of Jilin Province, according to the main data bulletin of the sixth national population census in $2010,{ }^{15}$ and therefore, the expected sample size was $3355,1 \%$ of the targeted population. The sample size (N) necessary for this cross sectional study was calculated based on a prevalence (p) of stroke of $2.37 \%$ among adults aged 40 years or older in China ${ }^{17}$ with a $0.5 \%$ uncertainty level (d), using the formula $n=t \alpha^{2} \mathrm{pq} / \mathrm{d}^{2}$ (where $\mathrm{t}=1.96, \alpha=95 \%$ for both sides; $\mathrm{q}=1-\mathrm{p}$ ). Using this equation, we estimated a required sample size of 3556 subjects. Considering an estimated lost to follow-up rate of $20 \%$, the planned sample size was $4445(3556 / 0.80)$. Finally, 4100 permanent residents aged 40 years or older who had lived in Dehui City of Jilin Province for more than 6 months volunteered to participate in the survey, with a response rate of $92.2 \%(4100 / 4445)$. For the purpose of the present analysis, 48 subjects were excluded due to missing values, giving a total of 4052 people included in this analysis.

\section{Data collection and measurement}

All data were collected by face to face interviews. The questionnaire included demographic characteristics (eg, gender, age, education level and employment), stroke related behavioural factors (eg, smoking, drinking, exercise habits and diet), personal and family medical history of stroke and chronic diseases (ie, hypertension, diabetes mellitus, dyslipidaemia and atrial fibrillation), and physical examination and laboratory testing (eg, height, weight, resting blood pressure, fasting blood glucose and lipid, and ECG).

Height and weight were measured according to a standardised protocol and technique, with participants wearing no shoes. Blood pressure was measured by trained professionals using an electronic sphygmomanometer (OMRON HEM-7200), and each participant rested for at least $20 \mathrm{~min}$ before measurements were taken. Participants were measured twice, and the average of the readings was taken. Blood samples were drawn from participants' antecubital veins for measuring fasting blood glucose (FBG), triglycerides (TG), total cholesterol (TC), low density lipoprotein cholesterol (LDL-C) and high density lipoprotein cholesterol (HDL-C). Blood samples were collected from subjects in the morning after an overnight fast (at least 8 hours) and transported to the 
same laboratory (Changchun Kingmed Centre for Clinical Laboratory Co Ltd.) under refrigeration, and then stored at $-20^{\circ} \mathrm{C}$. The laboratory finished the blood examinations within 8 hours after receiving the samples and provided daily quality control charts. All data were double entered and validated. In addition, all participants underwent ECGs for the detection of atrial fibrillation.

\section{Assessment criteria}

'Stroke' is defined by the WHO as 'rapidly developing clinical signs of focal (or global) disturbance of cerebral function, with symptoms lasting 24 hours or longer or leading to death, with no apparent cause other than of vascular origin'. Pathological subtypes of stroke included ischaemic stroke and haemorrhagic stroke. ${ }^{18}$ By definition, silent stroke and transient ischaemic attack were not included. The survey respondents with a history of stroke were asked to provide investigators with their paper based medical records (inpatient or outpatient records), which contained the patient history, physical examination and neurological imaging information, and at least one CT scan at stroke onset, which can accurately identify haemorrhagic stroke from ischaemic stroke.

The self-reporting of chronic diseases (hypertension, diabetes mellitus, dyslipidaemia and atrial fibrillation) was verified by field investigation. Eight stroke related risk factors were defined as follows: hypertension was defined as a self-reported history and/or the use of antihypertensive medication in the past 2 weeks, or the average of two resting systolic blood pressure (SBP) readings of $\geq 140 \mathrm{~mm} \mathrm{Hg}$ and/or diastolic blood pressure (DBP) $\geq 90 \mathrm{~mm} \mathrm{Hg}$ in the field survey. ${ }^{19}$ Dyslipidaemia was defined as using an antilipidaemic medication or having one or more of the following in the field survey: TG $\geq 1.70 \mathrm{mmol} / \mathrm{L}, \mathrm{TC} \geq 5.18 \mathrm{mmol} / \mathrm{L}$, HDL-C $<1.04 \mathrm{mmol} / \mathrm{L}$ and LDL-C $\geq 3.37 \mathrm{mmol} / \mathrm{L} .{ }^{20}$ Diabetes mellitus was defined as the use of insulin and/ or oral hypoglycaemic medications, or a self-reported history of diabetes or FBG $\geq 7.0 \mathrm{mmol} / \mathrm{L}$ in the field survey. ${ }^{21}$ Atrial fibrillation was defined as reported by the respondent or diagnosed by ECG in the field survey. ${ }^{22}$ A smoker was defined as someone who reported having smoked one or more cigarettes or was passively exposed to tobacco smoke every day for more than 6 consecutive months. A non-smoker was defined as never having smoked, and never having been passively exposed to tobacco smoke, or had history of smoking but quit smoking for at least 6 consecutive months previous to the study. ${ }^{15}$ Drinking status was divided into three categories according to the participants' self-reports in the previous 6 months: non-drinkers, light/moderate drinkers or heavy drinkers, according to the National Institute on Alcohol Abuse and Alcoholism (NIAAA) guidelines, advising limits of no more than 3 drinks per day or 7 drinks per week for men and women. These limits are based on a standard drink in the USA (i.e. any drink containing $14 \mathrm{~g}$ of pure alcohol). Using the NIAAA as a guideline, the following thresholds were applied: heavier drinkers, in excess of NIAAA limits and light/moderate drinkers, less than or up to the NIAAA limits. ${ }^{23}$

In addition, we grouped fruit consumption into three categories for our study: $\leq 2$ days per week ( $\leq 2$ days/week), 3-4 days/week and $\geq 5$ days/week. The weight of consumed fruits should reach or exceed 1 serving per day, with the definition of a serving calculated as $80 \mathrm{~g}$ of fruit. ${ }^{24}$ Body mass index (BMI) was calculated as weight $(\mathrm{kg})$ divided by height squared $\left(\mathrm{m}^{2}\right)$, and overweight or obesity was defined as BMI $\geq 26 \mathrm{~kg} / \mathrm{m}^{2} .{ }^{25}$ Lack of exercise was defined as physical exercise $<3$ times a week for $<30$ min each time, and this included industrial and agricultural labour. A family history of stroke was restricted to immediate family members.

Subjects with at least three of the aforementioned eight risk factors or a medical history of stroke were classified as the high risk stroke population. Subjects with fewer than three of these risk factors and with at least one of the three chronic diseases (ie, hypertension, diabetes mellitus and atrial fibrillation) were classified as the moderate risk stroke population. Subjects with fewer than three of these risk factors but without one of the three chronic diseases (ie, hypertension, diabetes mellitus and atrial fibrillation) were classified as the low risk stroke population. ${ }^{1526}$ The risk assessment scales for stroke referred were put forward by the Stroke Screening and Prevention Project Committee, National Health and Family Planning Commission of China, ${ }^{15}$ and have been proved to have good reliability and validity compared with the modified scale of the Framingham Stroke Profile (FSP), and can be used as an evaluation tool for stroke risk assessment in Chinese people. $^{27}$

\section{Statistical methods}

Taking into account the study's complex sampling design, the study data were weighted by gender (men or women), age group (40-49 years, 50-59 years, 60-69 years, $70+$ years) and place of residence (rural or urban area) according to the census data from Jilin Provincial Bureau of Statistics, to ensure that the study sample was best representative of the adult population (40+ years of age) in Dehui City of Jilin Province. The study sample was subdivided into 16 subgroups according to the above stratification factors. The formula for calculating the weighted value of each subgroup was as follows:

$$
\omega_{i}=\frac{\left(\sum n_{i}\right) C_{i}}{n_{i}}
$$

(where $\omega_{i}=$ the weighted value of each subgroup; $C_{i}=$ the constituent ratio of each subgroup in the overall; $n_{i}=$ the actual sample size of each group; $\sum n_{i}=$ the actual total sample size). The complex samples function of IBM SPSS 17.0 (SPSS Inc New York, New York, USA) was used for analysis. Continuous data are presented as means $\pm \mathrm{SD}$ and were compared using the Student's t test. Categorical variables are presented as proportions and were compared using the Rao-Scott $-\chi^{2}$ test between different subgroups. Estimates of prevalence rate, constituent ratio and their 
Table 1 Anthropometric characteristics of the study sample stratified by gender

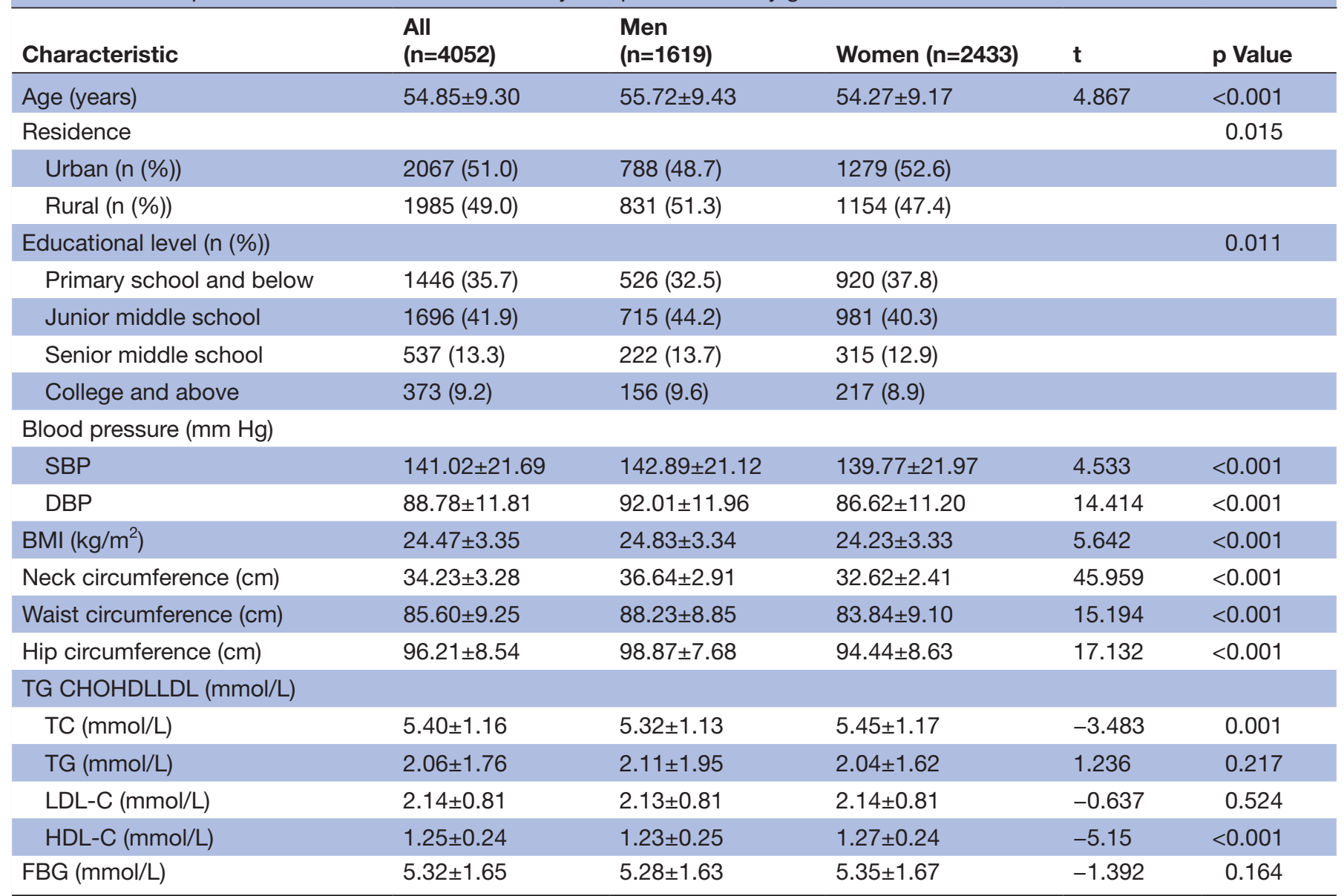

BMI, body mass index; DBP, diastolic blood pressure; FBG, fasting blood glucose; HDL-C, high density lipoprotein cholesterol; LDL-C, low density lipoprotein cholesterol; SBP, systolic blood pressure; TC, total cholesterol; TG, triglycerides.

95\% CIs for stroke and risk factors were generated by a range of characteristics, such as age group, gender, place of residence and level of education. Variables that were statistically significant at $\mathrm{p}<0.1$ in the univariate analysis were entered into the multivariate logistic regression analyses (cslogistic command in SPSS) to examine the variables independently associated with ischaemic and haemorrhagic stroke.

\section{RESULTS}

In total, 4052 participants (mean age $54.85 \pm 9.30$ years), 1619 men and 2433 women, were involved in the analysis. Nearly half $(49.0 \%)$ of the participants were from rural areas. Compared with women, men had a higher SBP (142.89 vs $139.77 \mathrm{~mm} \mathrm{Hg}$ ), DBP (92.01 vs $86.62 \mathrm{~mm} \mathrm{Hg}$ ), BMI (24.83 vs $\left.24.23 \mathrm{~kg} / \mathrm{m}^{2}\right)$, neck circumference (36.64 vs $32.62 \mathrm{~cm})$, waist circumference $(88.23$ vs $83.84 \mathrm{~cm})$ and hip circumference ( 98.87 vs $94.44 \mathrm{~cm}$ ) (all $\mathrm{p}<0.001$ ). The laboratory features of the participants by gender are listed in table 1.

As shown in table 2, the overall prevalence of stroke was $7.2 \%$ (95\% CI $6.3 \%$ to $8.2 \%$ ). The prevalence of stroke increased with age $(p<0.001)$, but decreased with educational level $(\mathrm{p}<0.001)$. It was significantly higher in men than in women $(8.8 \%$ vs $5.7 \%, \mathrm{p}=0.001)$. However, there was no statistically significant difference between rural and urban residents. Similar to the overall prevalence of stroke, the prevalence of ischaemic stroke also increased with age $(\mathrm{p}<0.001)$, but decreased with educational level $(p<0.001)$. In addition, the ischaemic stroke prevalence rate was higher among men than among women $(8.2 \%$ vs $5.2 \%, p=0.001)$, but no statistically significant difference was found between individuals in rural and urban areas.

As shown in table 3, of all the stroke cases, 91.7\% (95\% CI $87.4 \%$ to $94.6 \%)$ were ischaemic stroke and $8.3 \%(95 \%$ CI $5.4 \%$ to $12.6 \%)$ were haemorrhagic stroke. In addition, the proportions of different types of stroke showed no significant differences by age group, gender, residence or educational level. According to the different stroke risk levels, participants were divided into three groups, with over half $(57.3 \%, 95 \%$ CI $55.4 \%$ to $59.1 \%$ ) in the high risk stroke group. The proportions of the different stroke risk populations differed significantly by age group, gender, residence and educational level. 
Table 2 Prevalences of stroke and its subtypes by demographic characteristics

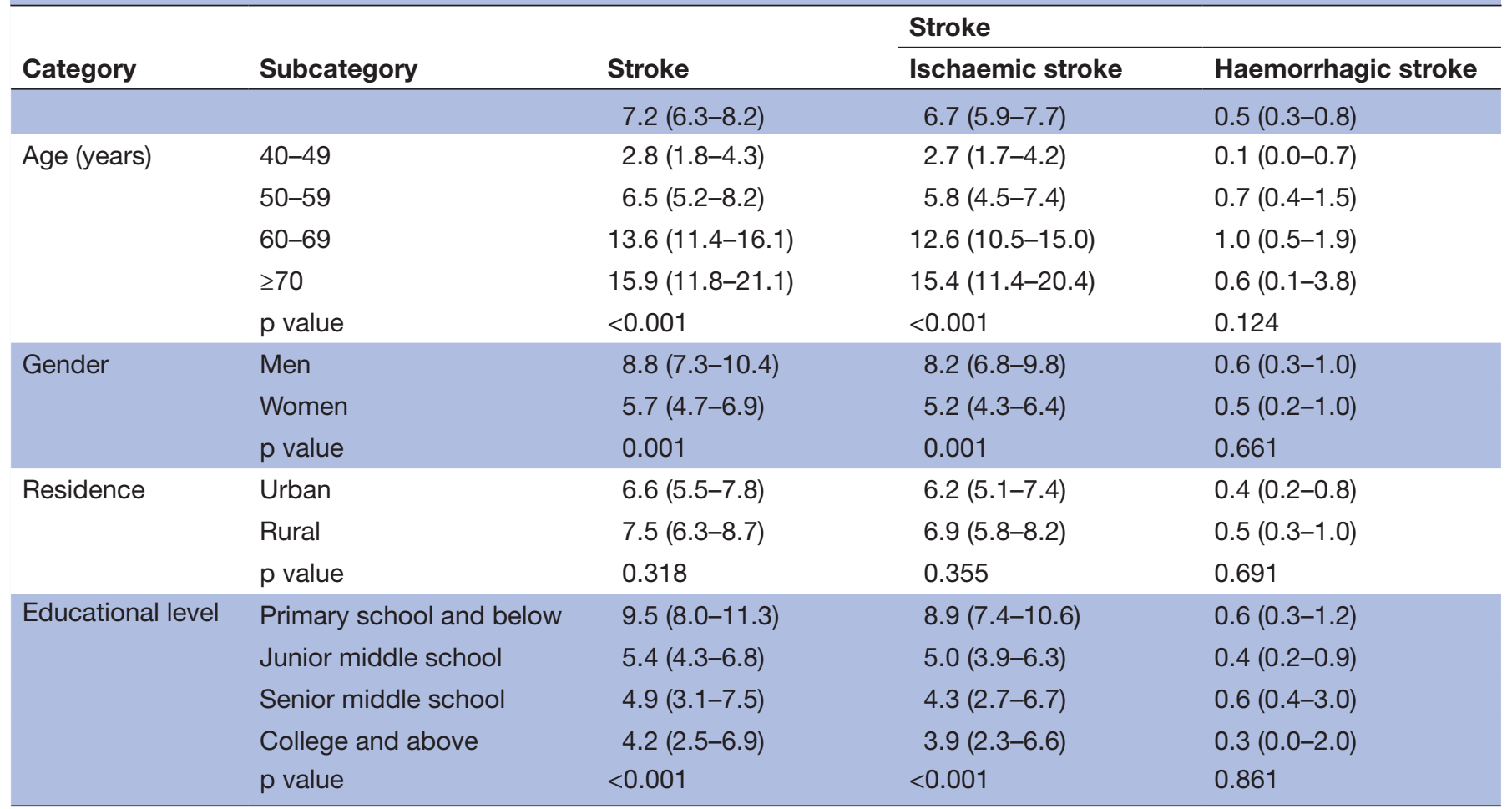

Data are presented as weighted prevalence $(95 \% \mathrm{Cls})$.

$p$ values were calculated with the Rao-Scott- $\chi^{2}$ test.

Table 4 describes the prevalence of stroke risk factors by demographic characteristics. The prevalence rates of hypertension, diabetes mellitus and atrial fibrillation increased with age $(\mathrm{p}<0.001)$; however, dyslipidaemia was more popular in adults aged $60-69$ years $(p<0.05)$. The prevalence rates of other stroke risk factors differed significantly by gender, but not by residence, except for dyslipidaemia, diabetes mellitus, smoking and lack of exercise. In addition, compared with the non-stroke group, we found that subjects with a history of stroke had a higher prevalence of hypertension, dyslipidaemia, diabetes mellitus, smoking, lack of exercise and family history of stroke $(p<0.001$, except $p<0.05$ for diabetes mellitus and smoking).

The multivariable logistic regression model indicated that multiple characteristics were positively associated with ischaemic stroke, including age, gender, hypertension, dyslipidaemia, lack of exercise and family history of stroke. Table 5 shows that the adjusted ORs of age groups $50-59,60-69$ and $\geq 70$ years (vs $40-49$ years age group) increased significantly with age $(\mathrm{p}<0.001)$, and men were more likely to have ischaemic stroke than women $(\mathrm{OR}=1.935,95 \%$ CI 1.410 to 2.655$)$. Patients with hypertension $(\mathrm{OR}=2.582,95 \% \mathrm{CI} 1.720$ to 3.877$)$ or dyslipidaemia $(\mathrm{OR}=1.513,95 \% \mathrm{CI} 1.064$ to 2.151$)$ were more likely to have ischaemic stroke. The ORs for lack of exercise and family history of ischaemic stroke were $1.446(95 \%$ CI 1.011 to 2.068 ) and 1.941 (95\% CI 1.424 to 2.646), respectively. However, there was no significant difference between participants with different educational levels, atrial fibrillation, diabetes mellitus, smoking or frequency of fruit and vegetable consumption. In contrast, as shown in table 6 , although the univariate analyses of risk factors showed that haemorrhagic stroke was associated with age, gender, hypertension and a high level of total cholesterol, the multivariate analyses model found that only hypertension $(\mathrm{OR}=4.064,95 \% \mathrm{CI} 1.358$ to 12.160$)$ was significantly associated with haemorrhagic stroke.

\section{DISCUSSION}

In this population based cross sectional study, we have identified a high prevalence of stroke, especially ischaemic stroke, and cerebrovascular associated risk factors among adults aged 40 years or older in northeast China. Also, we found that regions with a higher prevalence of hypertension, dyslipidaemia and lack of exercise were associated with a high prevalence of stroke.

The prevalence of stroke in Dehui City of Jilin Province was almost three times more than the nationwide findings. ${ }^{17}$ The prevalence of stroke increased with age, and this result was consistent with those of other studies. ${ }^{28}$ China's rapid economic development in the past three decades has also increased life expectancies, according to the World Bank, and the proportion of elderly people in the population has increased. The effect of population ageing on the prevalence of stroke has become more and more serious. Our study showed that the prevalence of stroke was significantly higher in men than in women, in accordance with past studies. ${ }^{132}$ However, in contrast 


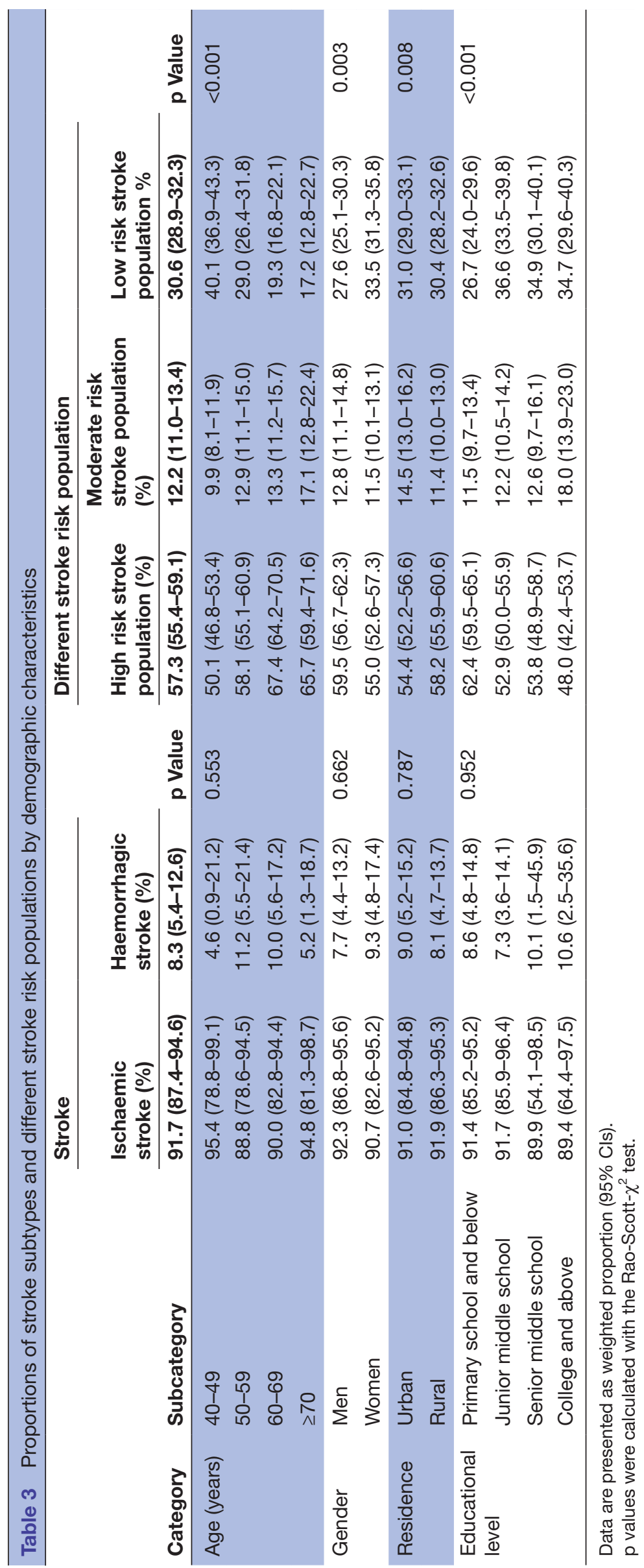




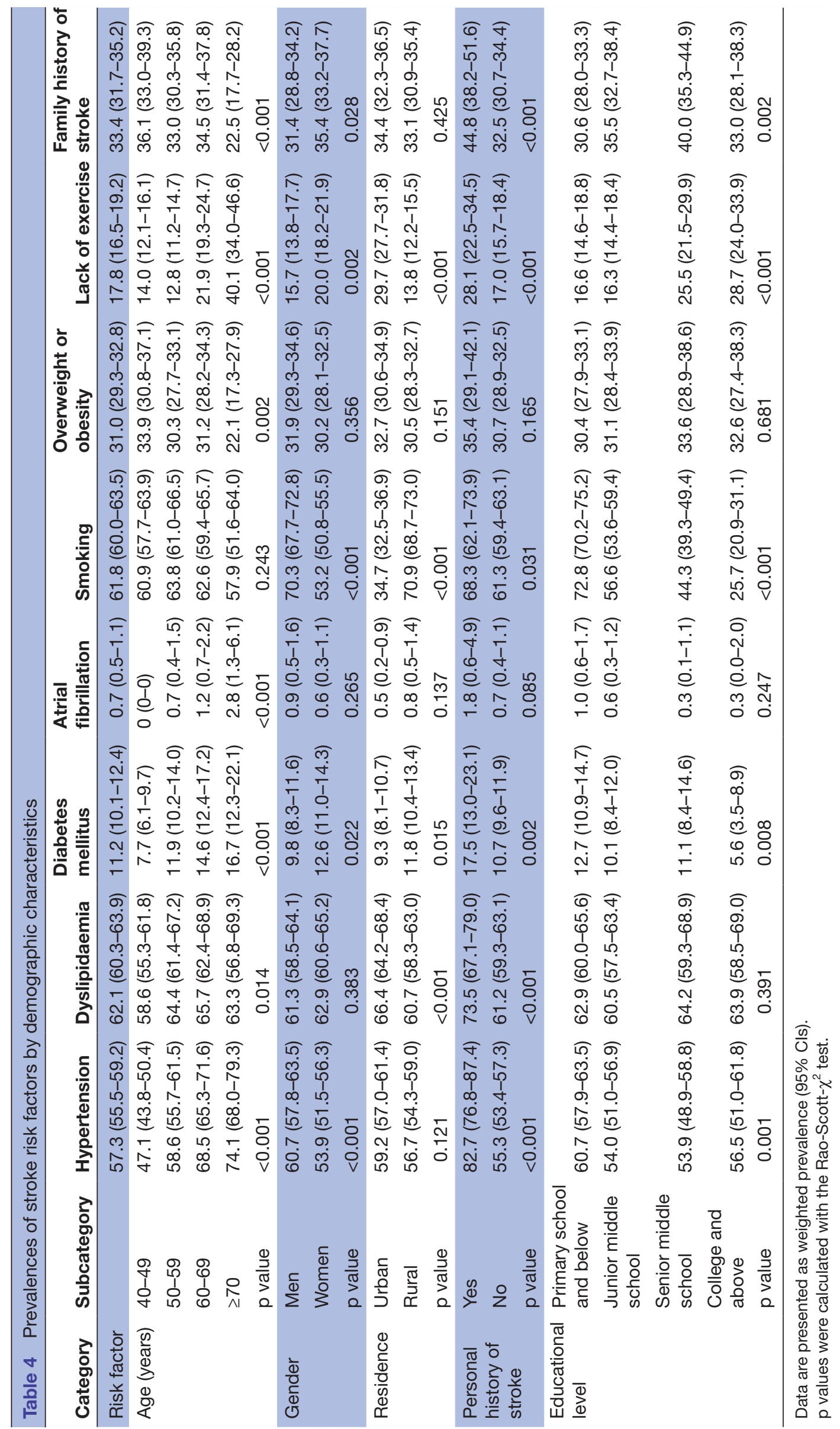


Table 5 Multivariate logistic analysis of the ischaemic stroke risk factors among participants

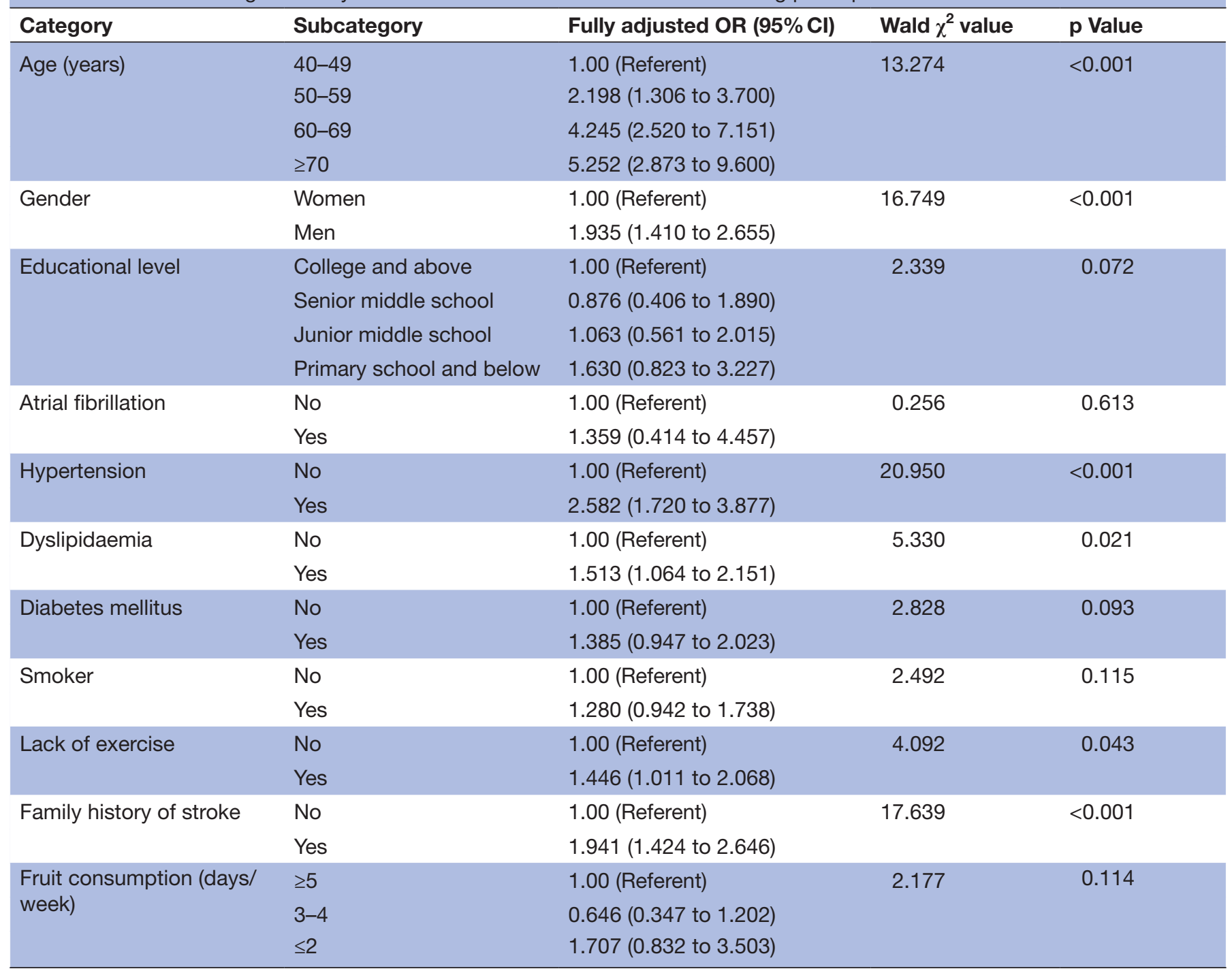

with a previous study, ${ }^{29}$ there was no statistically significant difference between rural and urban areas for stroke prevalence, suggesting disparities in residence was in line with rapid economic development.
Of all the stroke cases in our survey, $91.7 \%(95 \%$ CI $87.4 \%$ to $94.6 \%)$ were ischaemic stroke and $8.3 \%(95 \%$ CI $5.4 \%$ to $12.6 \%$ ) were haemorrhagic stroke. The proportion of ischaemic stroke was higher than those

\begin{tabular}{|c|c|c|c|c|}
\hline Category & Subcategory & Fully adjusted OR $(95 \% \mathrm{CI})$ & Wald $\chi^{2}$ value & p Value \\
\hline \multirow[t]{3}{*}{ Age (years) } & $40-49$ & 1.00 (Referent) & 1.696 & 0.166 \\
\hline & $60-69$ & 7.816 (1.266 to 48.240$)$ & & \\
\hline & $\geq 70$ & 3.835 (0.296 to 49.762$)$ & & \\
\hline Gender & $\mathrm{Me}$ & 1.597 (0.579 to 4.406$)$ & & \\
\hline \multirow[t]{2}{*}{ Hypertension } & No & 1.00 (Referent) & 6.290 & 0.012 \\
\hline & Yes & 4.064 (1.358 to 12.160$)$ & & \\
\hline High level of total cholesterol & No & 1.00 (Referent) & 2.674 & 0.102 \\
\hline
\end{tabular}


reported in developed countries, where ischaemic stroke accounted for approximately $67.3-80.5 \%$ of all stroke cases. ${ }^{30}$ This proportion was also markedly higher than that in three large cities in China in the 1990s. ${ }^{31}$ These findings were gathered from a prospective register and monitoring, in addition to annual retrospective door to door investigations, which found that cerebral infarction accounted for $43.7-78.9 \%$ of the total number of stroke cases. In addition, the proportion of stroke subtypes was quite different from those in Changsha, a city in south central China, where $55.4 \%$ of all stroke cases were attributed to intracerebral haemorrhage. ${ }^{32}$ This may reflect substantial geographic disparities and some underlying differences in dietary preferences and vascular risk factors. ${ }^{10}{ }^{33}$ However, this proportion was in accordance with the China Acute Cerebrovascular Events Register's (CACER-I) study, reporting that ischaemic stroke cases were increasing, whereas intracerebral haemorrhage showed a decreasing trend. ${ }^{34}$ The decrease in haemorrhagic stroke may be related to the improvement in hypertension control, as approximately $50 \%$ of acute haemorrhagic strokes can be attributed to hypertension in the Chinese population. ${ }^{8}$

In the present population based survey, we found that the prevalence rates of dyslipidaemia, smoking and hypertension were ranked as the top three cerebrovascular risk factors, with rates of $62.1 \%, 61.8 \%$ and $57.3 \%$, respectively. We found that the prevalence of dyslipidaemia was significantly higher than the national levels in a recent cross sectional study. ${ }^{35}$ Additionally, the prevalence of dyslipidaemia was higher among those in urban compared with rural areas. This result was consistent with the large cross sectional domestic survey conducted from January 2007 to October $2010,{ }^{35}$ and another regional study in China. ${ }^{36}$ This was mainly attributed to the higher total cholesterol intake among urban residents compared with those living in rural areas. ${ }^{37}$ By contrast, the prevalence of diabetes mellitus was higher among rural than urban residents. This was found from a pooled analysis of 56 eligible studies in China, ${ }^{38}$ and the reason was further explored in a future study. Also, the prevalence of hypertension in our study was much higher than the nationwide average $\mathrm{e}^{39}$ and than that found in some other regional studies, ${ }^{4041}$ but close to the recent study in the Sichuan Tibetan population. ${ }^{42}$ The high prevalence of dyslipidaemia and hypertension might be ascribed to dietary preferences and other lifestyle issues of residents in Jilin Province. The cold weather limits people's outdoor physical activity during the long winter, consequently increasing the risk of being overweight or obese and the related metabolic abnormalities. The present study also showed that the prevalence of smoking or being passively exposed to tobacco smoke, especially in rural areas, was higher than the rates from the China Global Adults Tobacco Survey in $2010 .^{43}$ This may be partly attributable to the fact that residents in Dehui City, in general, were undereducated $(77.5 \%$ of residents had a junior middle school and below educational level, as shown in table 1) and may lack knowledge of smoking hazards.

Estimates from the GBD 2013 suggested that approximately $94 \%$ of the Chinese burden of stroke is attributable to the combined effects of modifiable risk factors, and that most stroke cases are attributable to behavioural factors (ie, smoking, poor diet and low physical activity) and metabolic factors (ie, high SBP, high BMI, high FPG, high TC),${ }^{44}$ and these results were consistent with those of the INTERSTROKE study, ${ }^{45}$ in which approximately $86 \%$ of strokes could be attributed to nine potentially modifiable metabolic and behavioural risk factors. Thus it is of vital importance to promptly identify these modifiable metabolic and behavioural risk factors among residents to lessen the burden of stroke. In the present study, it was shown that hypertension, dyslipidaemia (metabolic risk factors) and lack of exercise (behavioural risk factor) were associated with ischaemic stroke $(\mathrm{p}<0.05)$ independently, which was consistent with several previous studies. ${ }^{46-50}$ Physical inactivity has been proved to be associated with an increased risk of stroke. ${ }^{51}$ The benefits appear to occur from a variety of types of activity, including leisure time physical activity, occupational activity, such as agricultural work, and walking. In the present study, the prevalence rate of lack of exercise was $17.8 \%$ (95\% CI $16.5 \%$ to $19.2 \%$ ) in total, and a difference in prevalence between urban and rural areas was noted, with urban being higher than rural areas $(29.7 \%$ vs $13.8 \%, \mathrm{p}<0.001)$. This may be attributed to a sedentary lifestyle among urban residents. The protective effect of physical activity may be partly mediated by its role in reducing blood pressure and controlling other risk factors for stroke, such as diabetes and excess body weight. ${ }^{52}{ }^{53}$ In addition, age, gender and family history of stroke were also risk factors for ischaemic stroke, which has been widely acknowledged nowadays. ${ }^{54}$ However, only hypertension $(\mathrm{OR}=4.064$, $95 \%$ CI 1.358 to 12.160 ) was significantly associated with haemorrhagic stroke in our survey when using the multivariate logistic regression model. That hypertension is an independent risk factor in the Chinese population has also been revealed in the Sino-MONICA-Beijing Project observational study. ${ }^{8}$

As far as we know, this was the most recent investigation of stroke and associated risk factors in this region, and it filled the information gap in this field. This cross sectional study involved a large representative sample of the Dehui City population. Strict training was done before the investigation, and we used a unified precoded questionnaire designed by the Stroke Screening and Prevention Programme of the National Health and Family Planning Commission of China. ${ }^{14}$ The data analysis was performed using complex weighted computation. For all the aforementioned reasons, our survey probably provided accurate data on the prevalence of stroke and related risk factors in this region. Some limitations of this study should be noted. Limitations of the study were the properties of the cross sectional study and the recall bias of the self-reported questionnaire. Another limitation was 
that the respondents' atrial fibrillation status was based on self-report and ordinary ECG, which may have underestimated paroxysmal atrial fibrillation. ${ }^{56}$

\section{CONCLUSIONS}

In conclusion, we have identified a high prevalence of stroke, especially ischaemic stroke, and associated risk factors among adults aged 40 years and older in northeast China. A higher regional prevalence of hypertension, dyslipidaemia and lack of exercise (modifiable risk factors) may be responsible, and these factors are appropriate targets for population based stroke prevention. Health policy makers should place more emphasis on the effective prevention and control of stroke and its risk factors.

Acknowledgements We gratefully acknowledge all study participants, interviewers from the First Hospital of Jilin University and the support from the Chinese National Centre for Stroke Care Quality Control and Management.

Contributors Conception and design: $Y-Q X$ and YY. Acquisition of the data: F-LZ, $\mathrm{H}-\mathrm{YL}$, YL and M-SS. Analysis: F-LZ and Y-HW. Drafting the manuscript: F-LZ. Critical revision: Z-NG, Y-HW and YY. All authors approved the final version to be published.

Funding This work was supported by Natural Science Foundation of China (NSFC, grant No. 8100945) and Changbai Mountain Scholars, Jilin Provincial Government to YY.

\section{Competing interests None declared.}

Ethics approval The study design was approved by the human ethics and research ethics committees of the First Hospital of Jilin University (approval No: 2015-R-250). Written informed consent was obtained from the participants in the survey.

Provenance and peer review Not commissioned; externally peer reviewed.

Data sharing statement The dataset supporting the conclusions of this article can be made available upon request.

Open Access This is an Open Access article distributed in accordance with the Creative Commons Attribution Non Commercial (CC BY-NC 4.0) license, which permits others to distribute, remix, adapt, build upon this work non-commercially, and license their derivative works on different terms, provided the original work is properly cited and the use is non-commercial. See: http://creativecommons.org/ licenses/by-nc/4.0/

(c) Article author(s) (or their employer(s) unless otherwise stated in the text of the article) 2017. All rights reserved. No commercial use is permitted unless otherwise expressly granted.

\section{REFERENCES}

1. Murray CJ, Barber RM, Foreman KJ, et al. Global, regional, and national disability-adjusted life years (DALYs) for 306 diseases and injuries and healthy life expectancy (HALE) for 188 countries, 1990-2013: quantifying the epidemiological transition. Lancet 2015;386:2145-91.

2. GBD 2013 Mortality and Causes of Death Collaborators. Global, regional, and national age-sex specific all-cause and causespecific mortality for 240 causes of death, 1990-2013: a systematic analysis for the Global Burden of Disease Study 2013. Lancet 2015;385:117-71.

3. Feigin VL, Forouzanfar MH, Krishnamurthi R, et al. Global and regional burden of stroke during 1990-2010: findings from the Global Burden of Disease Study 2010. Lancet 2014;383:245-55.

4. Powers WJ, Derdeyn CP, Biller J, et al. American Heart Association/ American Stroke Association focused update of the 2013 guidelines for the early management of patients with acute ischemic stroke regarding endovascular treatment: a guideline for healthcare professionals from the American Heart Association/American Stroke Association. Stroke 2015;46:3020-35.

5. Sacco RL, Benjamin EJ, Broderick JP, et al. American Heart Association prevention conference. IV. prevention and rehabilitation of stroke. Risk factors. Stroke 1997;28:1507-17.

6. Hankey GJ. Ischaemic stroke--prevention is better than cure. $J R$ Coll Physicians Edinb 2010;40:56-63.

7. Kim AS, Cahill E, Cheng NT. Global stroke belt: geographic variation in stroke burden worldwide. Stroke 2015;46:3564-70.

8. Zhao D, Liu J, Wang W, et al. Epidemiological transition of stroke in China: Twenty-one-year observational study from the Sino-MONICABeijing Project. Stroke 2008;39:1668-74.

9. CZ. The Third Nationwide Survey on Causes of Death. Beijing: The Peking Union Medical College Press, 2008.

10. Xu G, Ma M, Liu X, et al. Is there a stroke belt in China and why? Stroke 2013;44:1775-83.

11. Li ZS YQ, Chen SM, Shu Q, et al. Epidemiological survey of cerebrovascular disease in rural areas of China. Zhong-Hua ShenJing Wai-ke Za-Zhi 1989;5:7-11.

12. $\mathrm{Gx} \mathrm{W}, \mathrm{Zs} \mathrm{W}, \mathrm{BI} \mathrm{H}$. Epidemiological characteristics of stroke in 16 provinces of China. Zhonghua yi xue za zhi 1994;74:281:

13. Cheng X-M, Ziegler DK, Lai Y-HC, et al. Stroke in China, 1986 through 1990. Stroke 1995;26:1990-4.

14. Sun H, Zou X, Liu L. Epidemiological factors of stroke: a survey of the current status in china. J Stroke 2013;15:109-14.

15. Stroke Screening and Prevention Project Committee NHaFPCoC. Technical specification of stroke screening and prevention in China. Chinese Journal of the Frontiers of Medical Science 2013;9:44-50.

16. Feigin VL, Krishnamurthi RV, Parmar P, et al. Update on the global burden of ischemic and hemorrhagic stroke in 1990-2013: The GBD 2013 Study. Neuroepidemiology 2015;45:161-.

17. Li J, Wang L, Chao B, et al. Prevalence of stroke in China: an epidemiological study based on the National Stroke Screening Survey. The Lancet 2015;386:S49.

18. Aho K, Harmsen P, Hatano $S$, et al. Cerebrovascular disease in the community: results of a WHO collaborative study. Bull World Health Organ 1980;58:113-30.

19. James PA, Oparil S, Carter BL, et al. Evidence-based guideline for the management of high blood pressure in adults: report from the panel members appointed to the Eighth Joint National Committee (JNC 8). JAMA 20142014;311:507-20;311:507-20.

20. Joint Committee for Developing Chinese guidelines on Prevention and Treatment of Dyslipidemia in Adults. Chinese guidelines on prevention and treatment of dyslipidemia in adults. Zhonghua Xin Xue Guan Bing Za Zhi 2007;35:390-419.

21. American Diabetes Association. Diagnosis and classification of diabetes mellitus. Diabetes Care 2013;36:S67-74.

22. Fuster V, Rydén LE, Cannom DS, et al. ACC/AHA/ESC 2006 guidelines for the management of patients with atrial fibrillation: full text: a report of the American College of Cardiology/American Heart Association Task Force on practice guidelines and the European Society of Cardiology Committee for Practice Guidelines (Writing Committee to Revise the 2001 guidelines for the management of patients with atrial fibrillation) developed in collaboration with the European Heart Rhythm Association and the Heart Rhythm Society. Europace 2006;8:651-745.

23. Willenbring ML, Massey SH, Gardner MB. Helping patients who drink too much: an evidence-based guide for primary care clinicians. Am Fam Physician 2009;80:44-50.

24. He FJ, Nowson CA, MacGregor GA. Fruit and vegetable consumption and stroke: meta-analysis of cohort studies. Lancet 2006;367:320-6.

25. Rao W, Su Y, Yang G, et al. Cross-sectional associations between body mass index and hyperlipidemia among adults in northeastern China. Int J Environ Res Public Health 2016;13:516.

26. Mi T, Sun S, Zhang G, et al. Relationship between dyslipidemia and carotid plaques in a high-stroke-risk population in Shandong Province, China. Brain Behav 2016;6:e00473.

27. Shi Yu, WC-JRL JH. Study on reliability and validity of the risk assessment scale of stroke. Chinese Journal of Stroke 2016;11:202-6.

28. Li SC, Schoenberg BS, Wang CC, et al. Cerebrovascular disease in the People's Republic of China: epidemiologic and clinical features. Neurology 1985;35:1708-13.

29. Xue GB, Yu BX, Wang XZ, et al. Stroke in urban and rural areas of China. Chin Med J 1991;104:697-704.

30. Feigin VL, Lawes CM, Bennett DA, et al. Stroke epidemiology: a review of population-based studies of incidence, prevalence, and case-fatality in the late 20th century. Lancet Neurol 2003;2:43-53. 
31. Jiang $B$, Wang $W-z$, Chen $\mathrm{H}$, et al. Incidence and trends of stroke and its subtypes in China: results from three large cities. Stroke 2006;37:63-5.

32. Yang QD, Niu Q, Zhou YH, et al. Incidence of cerebral hemorrhage in the Changsha community. A prospective study from 1986 to 2000. Cerebrovasc Dis 2004;17:303-13.

33. He J, Klag MJ, Wu Z, et al. Stroke in the People's Republic of China: I. Geographic variations in incidence and risk factors. Stroke 1995;26:2222-7.

34. Wu L, Wang A, Wang $X$, et al. Factors for short-term outcomes in patients with a minor stroke: results from China National Stroke Registry. BMC Neurol 2015;15:253.

35. Pan L, Yang Z, Wu Y, et al. The prevalence, awareness, treatment and control of dyslipidemia among adults in China. Atherosclerosis 2016;248:2-9.

36. Wang S, Xu L, Jonas JB, et al. Prevalence and associated factors of dyslipidemia in the adult Chinese population. PLoS One 2011;6:e17326.

37. Pang $X H$, Jiao SF, Huang $L$, et al. Nutrition and health status of the Beijing residents. Zhonghua Yu Fang Yi Xue Za Zhi 2005;39:269-72.

38. Li MZ, Su L, Liang BY, et al. Trends in prevalence, awareness, treatment, and control of diabetes mellitus in mainland china from 1979 to 2012. Int J Endocrinol 2013;2013:753150.

39. Bundy JD, He J. Hypertension and related cardiovascular disease burden in China. Ann Glob Health 2016;82:227-33.

40. Wang Y, Zhang J, Ding Y, et al. Prevalence of hypertension among adults in remote rural areas of Xinjiang, China. Int $J$ Environ Res Public Health 2016;13:524.

41. Hu M, Wan Y, Yu L, et al. Prevalence, awareness, treatment, and control of hypertension and associated risk factors among adults in Xi'an, China: A cross-sectional study. Medicine 2016;95:e4709.

42. Huang X, Zhou Z, Liu J, et al. Prevalence, awareness, treatment, and control of hypertension among China's Sichuan Tibetan population: A cross-sectional study. Clin Exp Hypertens. 2016 ;38:457-63.

43. Yang GH. Global Adult Tobacco Survey (GATS) China 2010 Country Report. 2011

44. Feigin VL, Roth GA, Naghavi M, et al. Global burden of stroke and risk factors in 188 countries, during 1990-2013: a systematic analysis for the Global Burden of Disease Study 2013. Lancet Neurol 2016;15:913-24.

45. O'Donnell MJ, Xavier D, Liu L, et al. Risk factors for ischaemic and intracerebral haemorrhagic stroke in 22 countries (the INTERSTROKE study): a case-control study. Lancet 2010;376:112-23.

46. He J, Klag MJ, Wu Z, et al. Stroke in the people's Republic of China. II. Meta-analysis of hypertension and risk of stroke. Stroke 1995;26:2228-32.

47. Ebrahim S, Sung J, Song YM, et al. Serum cholesterol, haemorrhagic stroke, ischaemic stroke, and myocardial infarction: Korean national health system prospective cohort study. BMJ 2006;333:22.

48. Tanne D, Yaari S, Goldbourt U. High-density lipoprotein cholesterol and risk of ischemic stroke mortality. A 21-year follow-up of 8586 men from the Israeli Ischemic Heart Disease Study. Stroke 1997;28:83-7.

49. Soyama Y, Miura K, Morikawa Y, et al. High-density lipoprotein cholesterol and risk of stroke in Japanese men and women: the Oyabe Study. Stroke 2003;34:863-8.

50. Alevizos A, Lentzas J, Kokkoris S, et al. Physical activity and stroke risk. Int J Clin Pract 2005;59:922-30.

51. Lee CD, Folsom AR, Blair SN. Physical activity and stroke risk: a meta-analysis. Stroke 2003;34:2475-81.

52. Manson JE, Colditz GA, Stampfer MJ, et al. A prospective study of maturity-onset diabetes mellitus and risk of coronary heart disease and stroke in women. Arch Intern Med 1991;151:1141-7.

53. Blair SN, Kampert JB, Kohl HW, et al. Influences of cardiorespiratory fitness and other precursors on cardiovascular disease and all-cause mortality in men and women. JAMA 1996;276:205-10.

54. Sacco RL. Risk factors and outcomes for ischemic stroke. Neurology 1995;45:S10-14

55. Knottnerus ILH, Gielen M, Lodder J, et al. Family history of stroke is an independent risk factor for lacunar stroke subtype with asymptomatic lacunar infarcts at younger ages. Stroke 2011:42:1196-200.

56. Koga M, Yoshimura S, Hasegawa Y, et al. SAMURAI Study Investigators. Higher Risk of Ischemic Events in Secondary Prevention for Patients With Persistent Than Those With Paroxysmal Atrial Fibrillation. Stroke 2016;47:2582-8. 